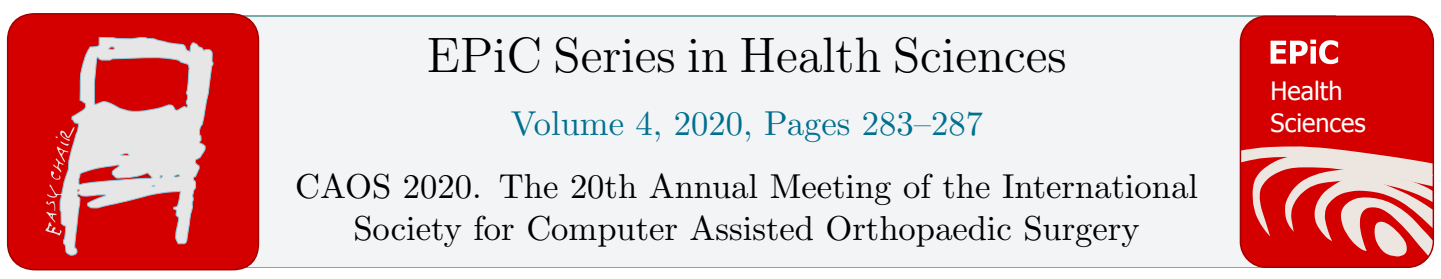

\title{
Intra-operative Soft Tissue Targets for Improved Outcomes in Total Knee Arthroplasty
}

\author{
Edgar A. Wakelin ${ }^{1 *}$, Sami Shalhoub ${ }^{1}$, Jeffrey M. Lawrence ${ }^{2}$, John M. \\ Keggi $^{3}$, Jeffrey H. DeClaire ${ }^{4}$, Amber L. Randall ${ }^{5}$, Corey E. Ponder ${ }^{6}$, Jan \\ Koenig $^{7}$, Christopher Plaskos ${ }^{1}$ \\ ${ }^{1}$ Corin Ltd., Raynham, MA, USA. \\ ${ }^{2}$ Gundersen Health System, Viroqua, WI, USA. \\ ${ }^{3}$ Connecticut Joint Replacement Institute, Hartford, CT, USA. \\ ${ }^{4}$ DeClaire LaMacchia Orthopaedic Institute, Rochester Hills, MI, USA. \\ ${ }^{5}$ Flagstaff Bone and Joint, Flagstaff, AZ, USA. \\ ${ }^{6}$ Oklahoma Sports and Orthopedics Institute, Edmond, OK, USA. \\ ${ }^{7}$ NYU Winthrop University Hospital, Long Island, NY, USA \\ edgar.wakelin@coringroup.com
}

\begin{abstract}
A poorly balanced, unstable or stiff joint is a leading cause of dissatisfaction and revision after surgery. The quantitative definition of a well-balanced joint, however, remains a source of controversy. This study investigates joint gaps measured by a digitally-controlled ligament tensioning device throughout flexion and its effect on postoperative outcome.

Surgeries were performed using the OMNIBotics robot-assisted TKA platform and BalanceBot device. Joint gaps were recorded by the BalanceBot throughout flexion during trialing. Knee Injury and Osteoarthritis Outcome Score (KOOS) was collected at 1-year post-op. Correlations between joint gaps and KOOS outcome were investigated.

Knees that reported: a medial gap $\leq$ lateral gap in extension $(\mathrm{p}=0.007$, median $\Delta=$ 8.3); an average joint gap of between $1 \mathrm{~mm}$ tighter and $1 \mathrm{~mm}$ looser than the final insert thickness in midflexion ( $\mathrm{p}=0.006$, median $\Delta=5.5$ ); and an imbalance of less than 1.5 $\mathrm{mm}$ in flexion ( $\mathrm{p}=0.012$, median $\Delta=2.8)$ reported significantly improved pain scores. Patients which satisfied both extension and flexion criteria, or midflexion and flexion criteria reported improved outcomes compared to those which satisfied only one or neither criterion ( $\mathrm{p}=0.0002$, median $\Delta=9.7, \mathrm{p}=0.0019$, median $\Delta=8.4$ respectively).

KOOS Pain scores correlated with joint gap measurements across all flexion angles investigated. Combining joint gap windows, subgroups of patients were found that reported a change in the median KOOS Pain outcome beyond the minimally clinically important difference.
\end{abstract}




\section{Introduction}

A poorly balanced, unstable or stiff joint is a leading cause of dissatisfaction and revision after surgery [1-4]. The quantitative definition of a well-balanced joint, however, remains a source of controversy [5]. Digital sensors provide a robust method of measuring joint balance intra-operatively [6] and previous studies have reported improved outcomes in knees that are more balanced throughout the range of motion [7]. However, the relationship between joint gap and balance measurements with patient outcome at different flexion angles has not yet been investigated.

This study investigates the joint gaps measured by a digitally-controlled ligament tensioning device throughout the range of motion and its effect on post-operative outcome. Our hypothesis is that joint gaps in extension, mid-flexion and full flexion correlate with one-year patient reported pain outcomes. Furthermore, we hypothesize that clinically relevant gap windows can be defined for targeting improved patient outcomes.

\section{Methods}

\subsection{Surgical Methods}

All surgeries were performed using the OMNIBotics robot-assisted TKA platform and BalanceBot device. The PCL was routinely resected and APEX implants were used in all cases implanting a CR femoral component with an Ultra-congruent tibial insert or using PS components. A mixture of tibiafirst gap balancing and femur-first (targeting mechanical alignment) approaches were performed. Surgeons were free to target any joint balance or gap profile. Medial and lateral joint gaps were recorded by the BalanceBot throughout the range of motion during trialing under an applied load of $70-90 \mathrm{~N}$ with the patella reduced. The thickness of the final tibial inserts implanted were then subtracted from the joint gap measurements to give final gap values, Figure 1. During data capture, the surgeon supported the femur posteriorly with the tibia in flexion. The knee was then extended with care not to apply a varus/valgus or internal/external rotation force to the joint.

\subsection{Outcome methods and analysis}

Knee Injury and Osteoarthritis Outcome Score (KOOS) was collected at 1-year post-op. Patients were asked to consider the past 7 days as a time frame for the performance of their knee. Correlations between joint gaps in Extension $\left(10^{\circ}\right)$, mid-flexion $\left(30^{\circ}-40^{\circ}\right)$ and full flexion $\left(>70^{\circ}\right)$ and $\mathrm{KOOS}$ outcome were performed using Spearman's correlation. Wilcoxon rank-sum tests were used to determine the difference between categorized gap data. All statistical analysis was performed using $\mathrm{R}$ 3.5.3. A total of 145 patients received TKA surgery using the BalanceBot device and completed 1year KOOS outcome scores.

\section{Results}

In extension, a significant correlation was found between the mediolateral (ML) gap difference and 1year pain outcome $\left(\mathrm{r}^{2}=-0.18, \mathrm{p}=0.033\right)$, in which knees with a balanced or tighter medial compartment reported improved pain outcomes. Knees with a medial gap $\leq$ lateral gap reported significantly improved pain scores $(\mathrm{p}=0.007$, median $\Delta=8.3$ ). In mid-flexion a significant correlation was found between the medial joint gap and 1 -year pain outcome $\left(\mathrm{r}^{2}=-0.18, \mathrm{p}=0.038\right)$ whereby a greater gap correlated with worse pain. Knees that reported an average joint gap of between $1 \mathrm{~mm}$ tighter and 1 $\mathrm{mm}$ looser than the final insert thickness reported significantly improved pain scores $(\mathrm{p}=0.006$, median $\Delta=5.5$ ). In flexion a significant correlation was found between the absolute value of the ML gap difference and 1-year pain outcome $\left(r^{2}=-0.20, p=0.016\right)$ whereby a greater imbalance correlated 
with worse pain. An imbalance of less than $1.5 \mathrm{~mm}$ reported significantly improved pain scores $(\mathrm{p}=$ 0.012 , median $\Delta=2.8$ ).

Patients which satisfied both extension and flexion criteria reported significantly improved outcomes compared to those which satisfied only one or neither criterion ( $\mathrm{p}=0.0002$, median $\Delta=9.7)$, Figure 2 . Similarly, patients which satisfied both midflexion and flexion criteria reported significantly improved outcomes compared to those which satisfied only one or neither criterion $(\mathrm{p}=0.0019$, median $\Delta=8.4$ ), Figure 2 .

\section{Discussion}

This study showed significant correlations between joint gaps measured in extension, mid-flexion and flexion and KOOS scores at 1-year. KOOS Pain scores correlated with joint gap measurements across all flexion angles investigated and allowed clinically achievable windows to be defined to identify outcomes that were statistically significantly improved. Combining joint gap windows in extension, mid-flexion and flexion, subgroups of patients were found that reported a clinically significant change in the median KOOS Pain outcome beyond the minimally clinically important difference [8].

Greater laxity in the lateral compartment in extension has been reported in a cadaver study by Roth et al. [9], similar to the present study. Increased native laxity and asymmetry of laxity, however, was reported by Roth and others [10] in contrast to the mid-flexion and flexion windows for improved outcomes found here. This may be due to the increasing contribution of the posterior cruciate ligament (PCL) in flexion which was resected in all knees here.

There are a number of limitations to this study. It is unknown if the ideal distraction force for TKA is between $70-90 \mathrm{~N}$. It is possible that the ideal force is patient specific and based on the individual's ligament properties. The force used here was chosen as it has previously been demonstrated to be safe clinically [11, 12], and assessed by experienced Orthopaedic Specialists to result in suitable postoperative stability [13]. Greater distraction force, however, may result in an overly tight joint and externally rotated femoral component when using a gap balancing technique [14].

Gap data investigated here is not normalized for patient specific anatomic, demographic or preoperative PROMS data. Previous literature has reported a number of pre-operative factors that can affect and be used to predict patient outcome [15-18]. Major demographic factors such as age, gender and BMI were not found to be significantly different between the joint gap groups identified. The gap windows identified here, therefore, can be interpreted as population-wide targets rather than patientspecific targets.

Future data collection is needed to investigate the effect of joint balance on outcome while taking in to account more patient-specific factors.

\section{References}

1. Hughes, R.E., H. Zheng, and B.R. Hallstrom, Michigan Arthroplasty Registry Collaborative Quality Initiative (MARCQI) Report: 2012-2017. University of Michigan, Ann Arbor, 2018.

2. $\quad$ Australian Orthopaedic Association National Joint Registry Annual Report 2019. 2019.

3. National Joint Registry (UK). 2018.

4. $\quad$ Swedish Knee Arthroplasty Registry, Annual Report 2018. 2018.

5. Hirschmann, M.T., et al., Alignment in TKA: what has been clear is not anymore! 2019, Springer.

6. Woon, C.Y.L., et al., Dynamic sensor-balanced knee arthroplasty: can the sensor "train" the surgeon? Arthroplasty Today, 2019. 5(2): p. 202-210. 
7. Golladay, G.J., et al., Are Patients More Satisfied with a Balanced TKA? The Journal of Arthroplasty, 2019.

8. Roos, E. KOOS FAQs. 2019 [cited 2019 Dec 13]; Available from:

http://www.koos.nu/index.html.

9. Roth, J.D. and S.M. Howell, Soft tissue balance of the native knee provides guidance for balancing a total knee arthroplasty, in Soft Tissue Balancing in Total Knee Arthroplasty. 2017, Springer. p. 17-27.

10. Gladnick, B.P., et al., Primary and coupled motions of the native knee in response to applied varus and valgus load. The Knee, 2016. 23(3): p. 387-392.

11. Higuchi, H., et al., Relationship between joint gap difference and range of motion in total knee arthroplasty: a prospective randomised study between different platforms. International orthopaedics, 2009. 33(4): p. 997-1000.

12. Nagai, K., et al., Soft tissue balance changes depending on joint distraction force in total knee arthroplasty. The Journal of arthroplasty, 2014. 29(3): p. 520-524.

13. A., G.K., Soft-tissue and alignment correction. The Bone \& Joint Journal, 2014. 96B(11_Supple_A): p. 78-83.

14. Nagai, K., et al., The Influence of Joint Distraction Force on the Soft-Tissue Balance Using Modified Gap-Balancing Technique in Posterior-Stabilized Total Knee Arthroplasty. The Journal of Arthroplasty, 2017. 32(10): p. 2995-2999.

15. Lungu, E., et al., Prediction of poor outcomes six months following total knee arthroplasty in patients awaiting surgery. BMC musculoskeletal disorders, 2014. 15(1): p. 299.

16. Twiggs, J.G., et al., Clinical and Statistical Validation of a Probabilistic Prediction Tool of Total Knee Arthroplasty Outcome. The Journal of Arthroplasty, 2019.

17. Halawi, M.J., et al., Patient Dissatisfaction After Primary Total Joint Arthroplasty: The Patient Perspective. The Journal of arthroplasty, 2019.

18. Van Onsem, S., et al., A new prediction model for patient satisfaction after total knee arthroplasty. The Journal of arthroplasty, 2016. 31(12): p. 2660-2667. e1.
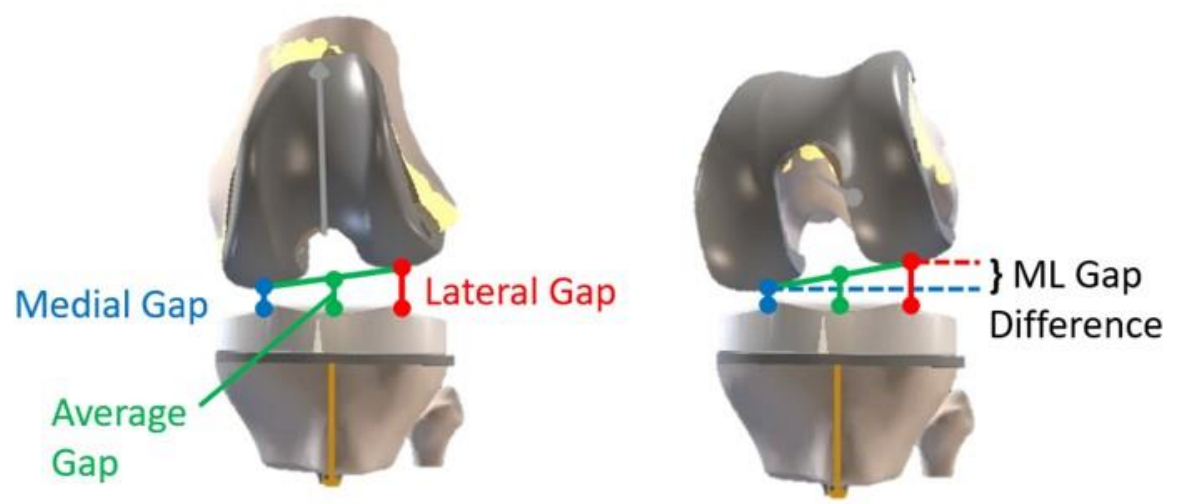

Figure 1 Joint gaps investigated 

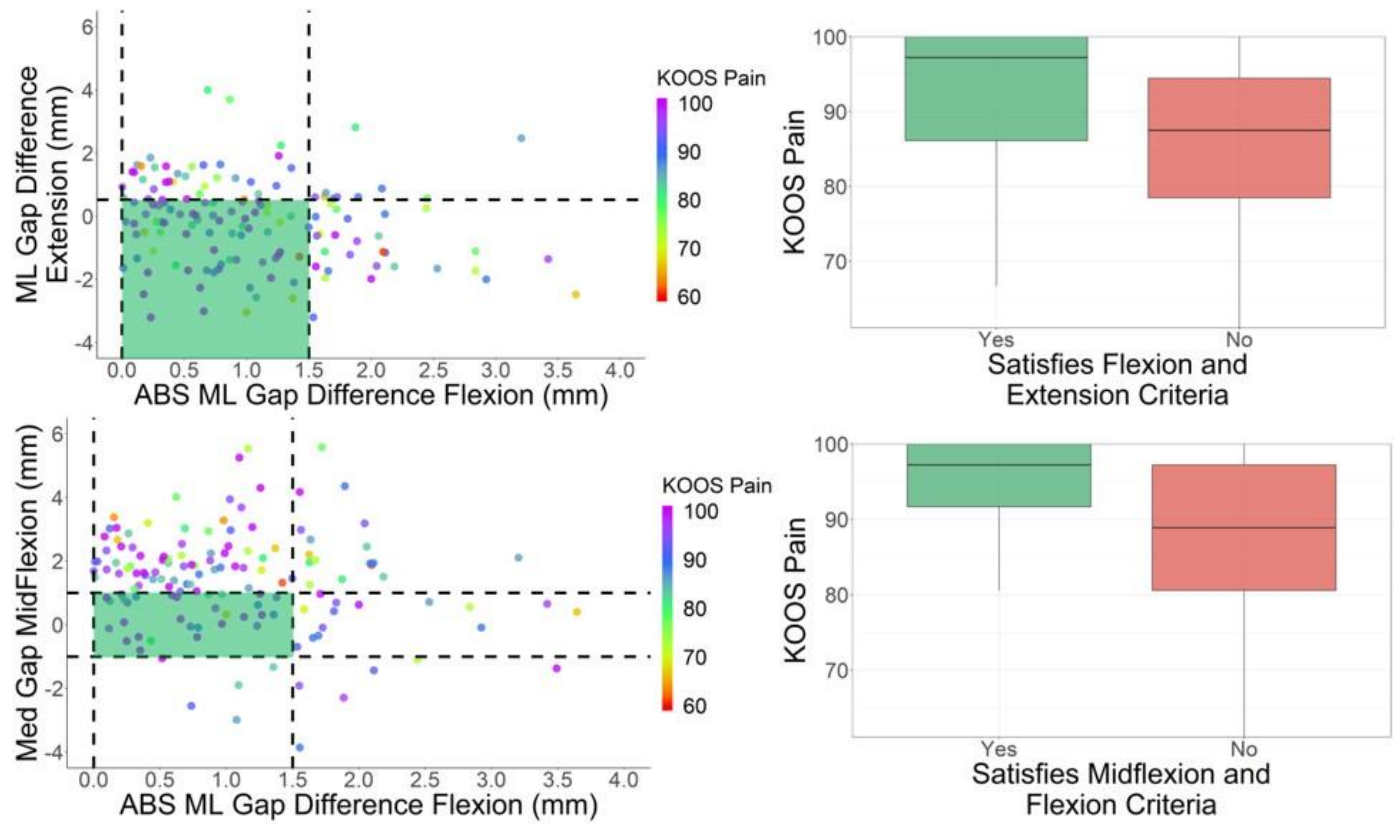

Figure 2 Top: Combination of Flexion and Extension balance targets for improved outcomes, $(p=0.0002$, median $\Delta=9.7)$. Bottom: Combination of Flexion and Midflexion balance targets for improved outcomes, $(p=$ 0.0019 , median $\Delta=8.4$ ). 